\title{
Splenic Liquefaction after Splenic Artery Embolization
}

\author{
Byung Hee Kang \\ Department of Trauma Surgery, Ajou University School of Medicine, Suwon, Korea
}

A 34-year-old man was transferred from a local hospital to our trauma center after a motor vehicle crash. He was diagnosed with a splenic laceration with bleeding (Figure 1). Multiple rib fractures with hemopneumothorax, liver laceration, cervical vertebral fracture, and thoracic vertebral fracture were also diagnosed. His blood pressure was 114/74 $\mathrm{mmHg}$ and heart rate was 110/min; he was intubated at the local hospital because of the lung injury. His vital signs were stable but systolic blood pressure dropped progressively to $100 \mathrm{mmHg}$, therefore splenic angiography was decided. No definite bleeding focus was identified, but venous or parenchymal bleeding from spleen injury was suspected. Hence, diffusion of the gelfoam particle embolization of the distal branch was performed to decrease the splenic perfusion (Figures 2,3). The next day, abdominal computed tomography (CT) revealed diffuse splenic ischemia (Figure 4). The patient was stabilized and extubated. He complained of left chest wall pain, but it could not be distinguished from the multiple rib fractures. He was moved to the general ward and the other fracture site was treated with an orthosis. Eleven days postembolization, CT revealed splenic liquefaction (Figure 5). Because his condition was stable, he was discharged and CT after 2 months showed atrophied spleen (Figure 6).

Splenic embolization is useful in blunt splenic injury, but it had several complication in-

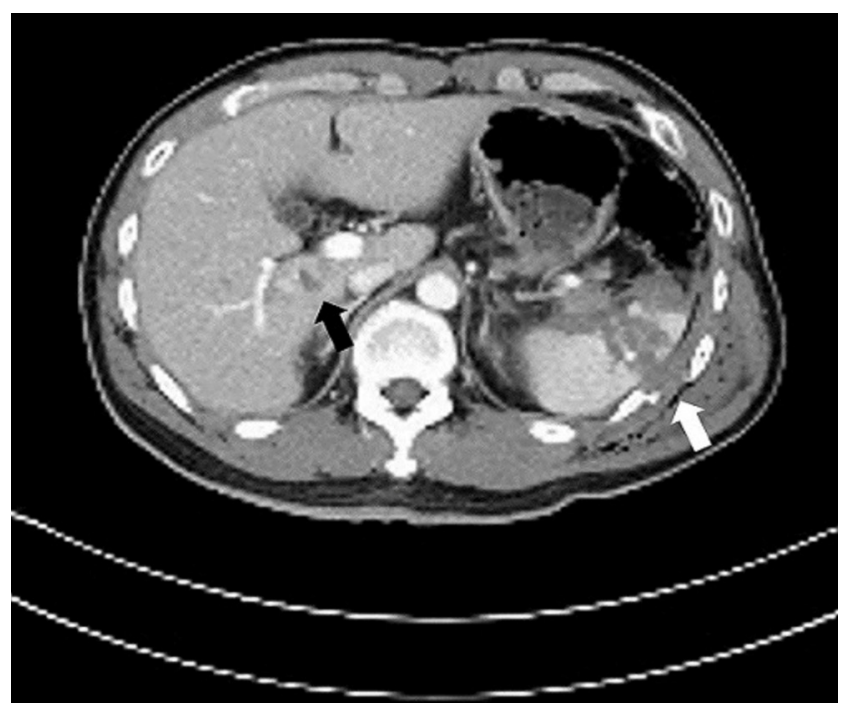

Figure 1. Spleen laceration with bleeding and rib fracture were presented (white arrow). Liver laceration was also presented (black arrow).

Copyright @ 2019 The Korean Society of Critical Care Medicine

This is an Open Access article distributed under the terms of Creative Attributions Non-Commercial License (http:// creativecommons.org/li-censes/by-nc/4.0/) which permits unrestricted noncommercial use, distribution, and reproduction in any medium, provided the original work is properly cited. 


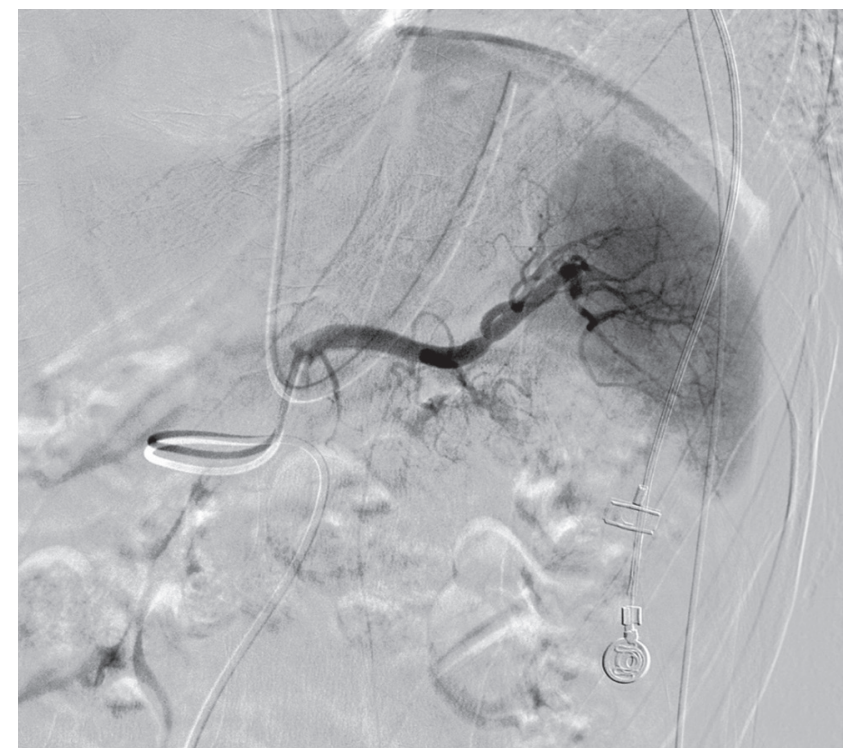

Figure 2. Definite bleeding was not identified in angiographic evaluation.

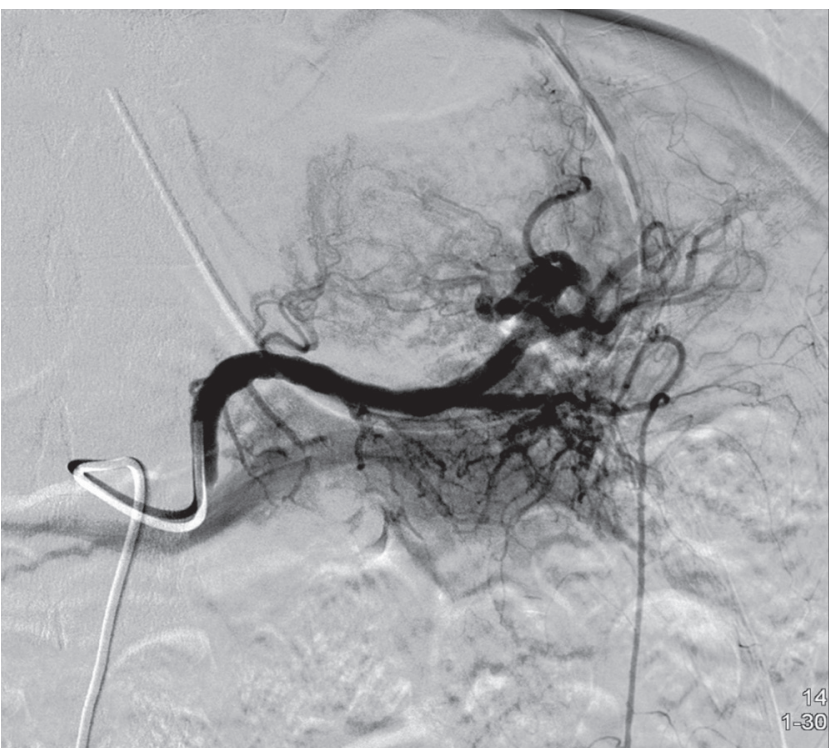

Figure 3. Diffuse distal branch embolization was performed to decrease splenic perfusion.

cluding splenic abscess, pseudocyst, pancreatitis, intestinal perforation, acute respiratory distress syndrome and infarction [1]. However, splenectomy is not usually required for these complications included splenic infarction. Moreover, $\mathrm{Li}$ et al. [2] reported that total splenic artery embolization for splenic artery aneurysms was safe and feasible. However, like in our case, follow-up CT could reveal unusual complications.

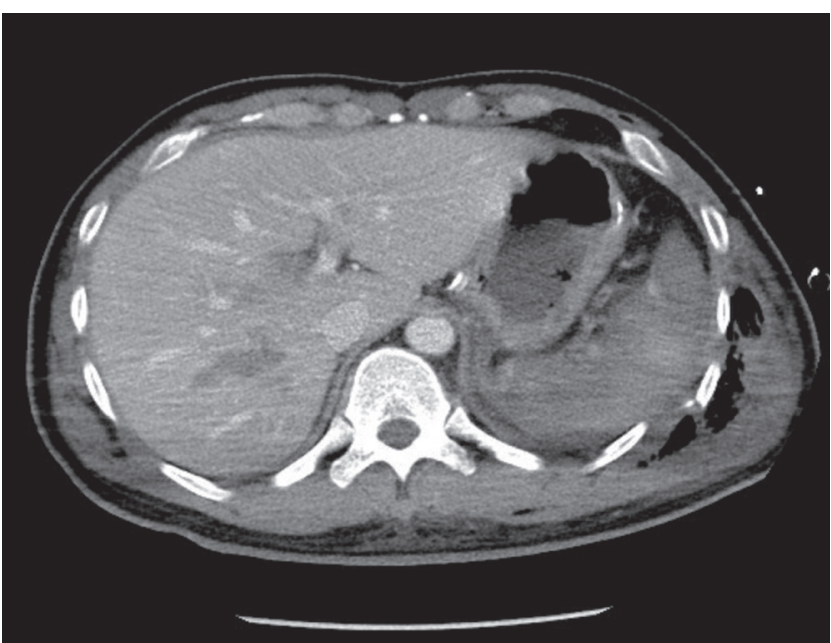

Figure 4. Spleen was not enhanced compared to liver.

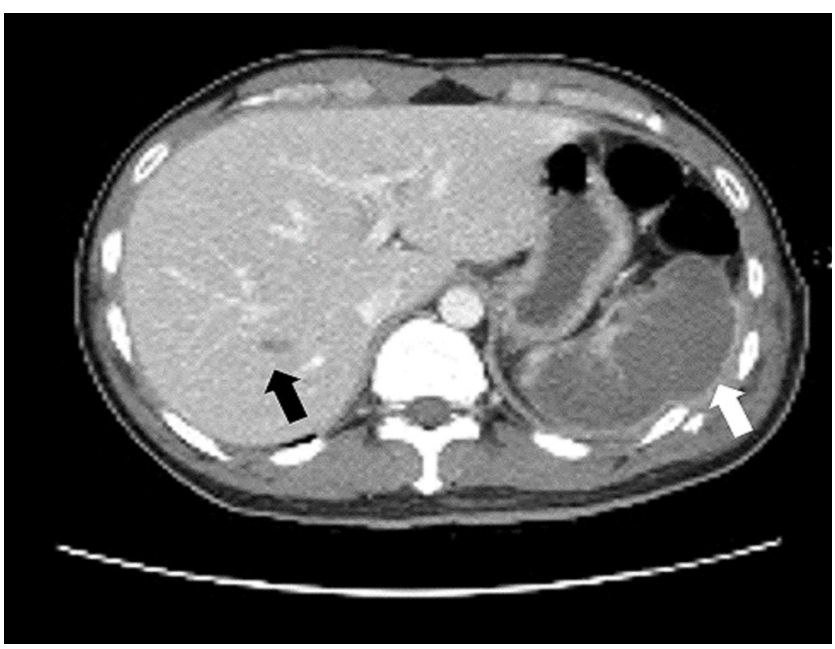

Figure 5. Eleven days post-embolization, splenic liquefaction was presented (white arrow). Liver laceration was improved (black arrow).

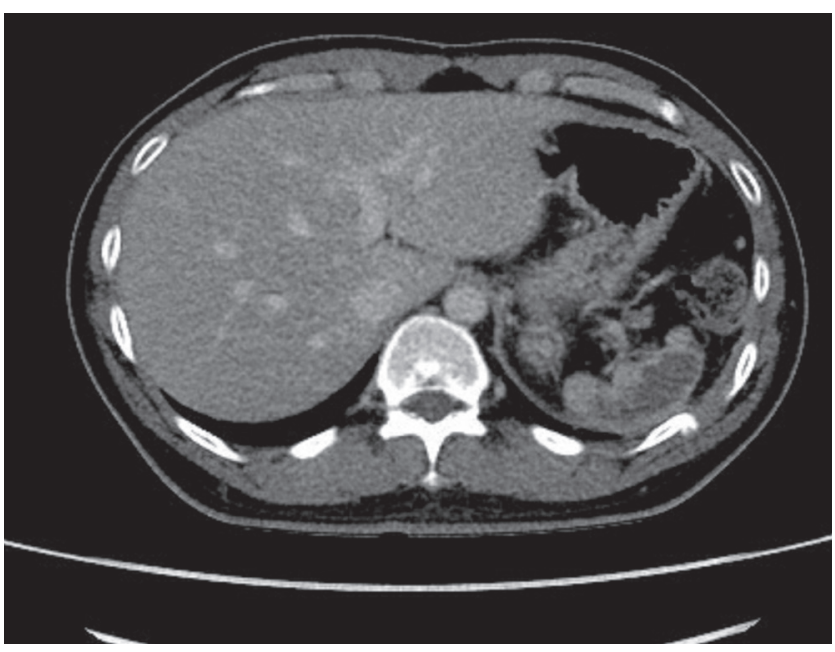

Figure 6. Spleen was atrophied after 2 month from embolization. 


\section{CONFLICT OF INTEREST}

No potential conflict of interest relevant to this article was reported.

\section{ORCID}

Byung Hee Kang

https://orcid.org/0000-0003-3204-3251

\section{REFERENCES}

1. Crichton JC, Naidoo K, Yet B, Brundage SI, Perkins Z. The role of splenic angioembolization as an adjunct to nonoperative management of blunt splenic injuries: a systematic review and meta-analysis. J Trauma Acute Care Surg 2017;83:934-43.

2. Li ES, Mu JX, Ji SM, Li XM, Xu LB, Chai TC, et al. Total splenic artery embolization for splenic artery aneurysms in patients with normal spleen. World J Gastroenterol 2014;20:555-60. 